\title{
Modelling and Design of HF RFID Passive Transponders with Additional Energy Harvester
}

\author{
Piotr Jankowski-Mihułowicz, Włodzimierz Kalita, \\ Mariusz Skoczylas, and Mariusz Węglarski \\ Department of Electronic and Communications Systems, Rzeszów University of Technology, W. Pola 2, 35-959 Rzeszów, Poland \\ Correspondence should be addressed to Piotr Jankowski-Mihułowicz; pjanko@prz.edu.pl
}

Received 22 May 2013; Accepted 12 August 2013

Academic Editor: Yuan Yao

Copyright (c) 2013 Piotr Jankowski-Mihułowicz et al. This is an open access article distributed under the Creative Commons Attribution License, which permits unrestricted use, distribution, and reproduction in any medium, provided the original work is properly cited.

\begin{abstract}
The huge progress in electronics technology and RFID technique gives the opportunity to implement additional features in transponders. It should be noted that either passive or semipassive transponders are supplied with energy that is derived from the electromagnetic field generated by the read/write device and its antenna. This power source is used to conduct radio-communication process and excess energy could be used to power the extra electronic circuits, but the problem is to determine the additional power load impact on the RFID system proper operation and size of interrogation zone. The ability to power the supplementary electronic blocks applied in the HF passive transponders is discussed in detail this paper. The simulation model and test samples with a harvester that recovers energy from the electromagnetic field of read/write device and its antenna have been developed in order to conduct investigations. The harvested energy has been utilized to supply a microprocessor acquisition block for LTCC pressure sensor developed in research previously described by authors.
\end{abstract}

\section{Introduction}

The (radio frequency identification) RFID technique is generally used in the processes of object identification. The permanent cost reduction of a single transponder and standardizations of operating conditions for all involved devices affect the broad implementation of these solutions in security and access control systems, industrial logistics (material supply or goods shipment), identification of measurement samples or valuable materials (in various areas of science, technology, or medicine), and forth [1-5]. Further improvements are feasible in many cases thanks to great achievements in modern electronics and significant progress in new technology of hybrid microelectronic circuits [6-8]. For example, it gives the opportunity to implement additional features in transponders. The extra functional blocks are usually powered by a built-in supply source-disposable battery. Unfortunately, since the batteries are used, the costs of applications with such transponders are very high and system maintenance is inconvenient with respect to totally passive solutions. However, it should be noted that either passive or semipassive transponders are supplied with energy that is derived from the electromagnetic field generated by the read/write device (RWD). This power source is used to conduct a radio-communication process and excess energy can be used to power the extra electronic circuits. But the problem is to determine the additional power load impact on the RFID system proper operation and size of interrogation zone (IZ).

The necessity to integrate a pressure sensor made in (low temperature cofired ceramic) LTCC technology [9] with a passive transponders consisted not only of a chip and antenna but also of a radio-frequency (RF) energy harvesting circuit (Figure 1) has been the key impulse to conduct the presented research.

The considerations also include interrogation zone (IZ) determination problems. The IZ is a space around the RWD where communication and energy conditions are met $[6,10$, 11]. Its shape and size is determined by the possibility of performing two tasks: providing transponders with the correct power supply and, as a result, establishing conditions for data radio communication with the RWD. It is the main parameter 


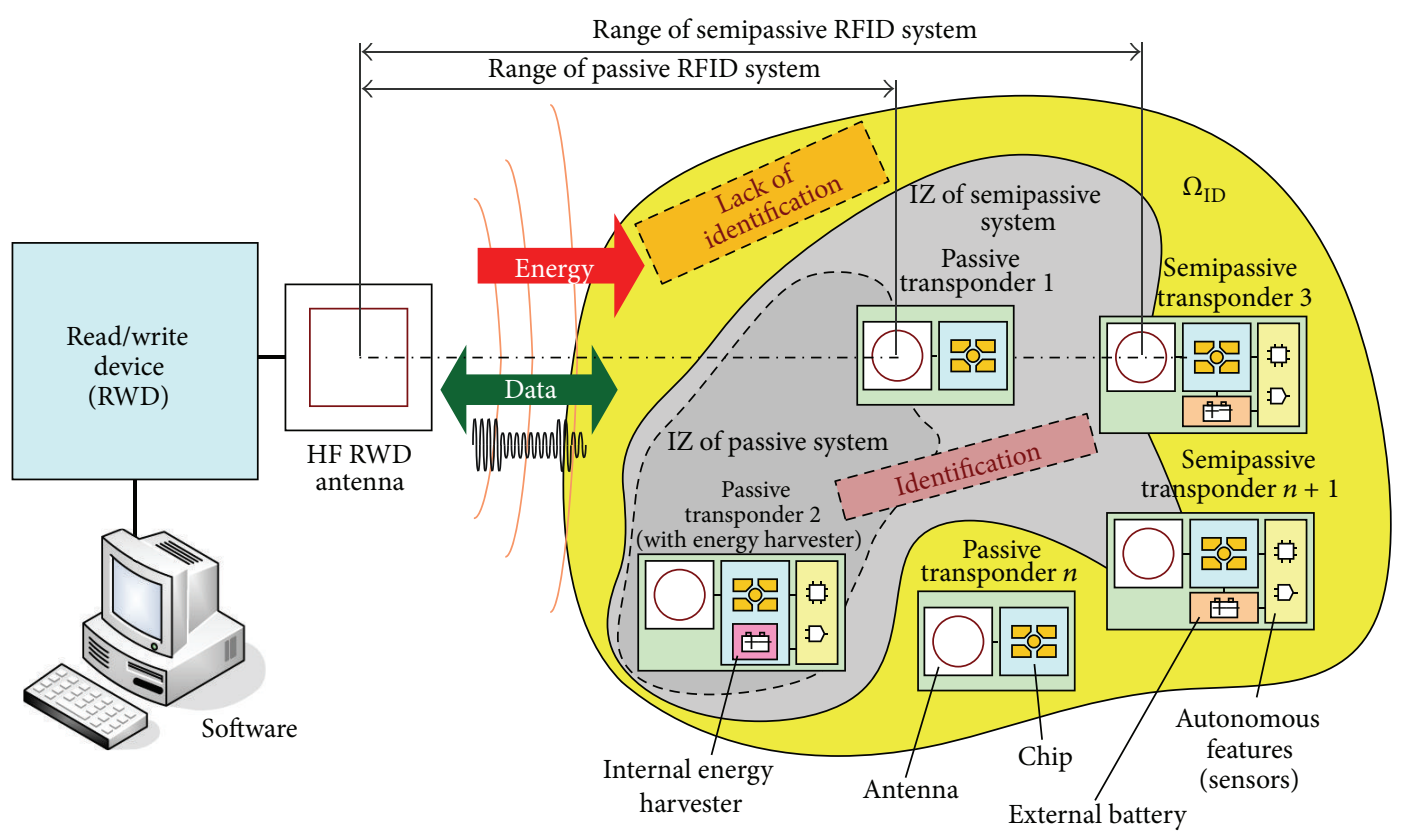

FIGURE 1: Block diagram of passive and semipassive RFID system.

because it comprehensively covers the matter of energy and communication activity of all RFID system parts. Since the interrogation zone is determined for the whole application (not for a single device), a knowledge base about essential properties of RFID equipment is necessary. But unfortunately, producers very often do not specify some essential electrical and structural parameters for their products. Because of this, the calculation of basic parameters describing interrogation zone (e.g., the maximal distance/range between transponder and the RWD antenna centre) is impossible. At the present stage of knowledge, it is the main reason why the practical implementation of anticollision identification is restrained especially in automated systems operating in dynamic conditions. In this situation, inefficient and timeconsuming trial and error methods are commonly used during system configurations. It does not provide reliable information on the operation and efficiency of the automated identification processes. The problems of RFID application predictability become even more important in the context of placing the physical quantity sensors in battery-less passive transponders.

The problem is further complicated in anticollision systems. The main characteristic of the single identification is the possibility to recognize only one object labelled with a radio transponder that has to be alone in the interrogation zone. In the case of the anticollision system, algorithms of multiaccess to radio channel are used and the communication process is carried out simultaneously with several transponders [10]. This makes it possible to automatically distinguish many objects appearing in the IZ at the same time. In both identification systems, it is assumed that labelled objects are present in the operating zone $\Omega_{\mathrm{ID}}$ (Figure 1), but there is no certainty that they will be recognized. The situation is even more complicated when the dynamic processes (with variable location and/or orientation of object in space) will be analyzed instead of the stationary one (with fixed location and orientation of objects in space) [12].

In RFID systems, the transponders are supplied with energy deriving from the electromagnetic field generated by the RWD. This supply source is always used to conduct a radio-communication process. But in transponders with sensors (sensors with RFID interface), it is necessary to provide energy for powering additional functional blocks.

The semipassive transponders (Figure 1) have a built-in extra source (e.g., lithium battery) which can be exchangeable or not [10]. A part of additional energy can be utilized to enlarge the size of interrogation zone but most of it is used for powering blocks of additional autonomous functions, such as measurement of physical quantities (humidity [13-15], temperature [16-19], light intensity [18], pressure [20], acceleration [21], gas [22], etc.), and writing gathered data in a builtin memory. These extra functions are carried out without the participation of RWDs. Because of battery, these types of transponders are more expensive (with respect to totally passive solutions) and have limited durability and there is necessity to replace worn out batteries. According to practice rules of RFID system usage, there is necessity to mechanically protect the batteries against thefts or to utilize disposable solutions (such transponders have to be replaced when the battery is exhausted or spoiled). However, it should be emphasized that the RWD has to be still active for properly conducting radio communication process, because the extra battery system can never be used for activating the transmitting circuit. It means that the antenna of transponder does not emit the electromagnetic field as it is in the case of conventional short range devices (SRD) $[23,24]$.

Disadvantages of the above mentioned power supply, development in the branch of new materials, and availability 


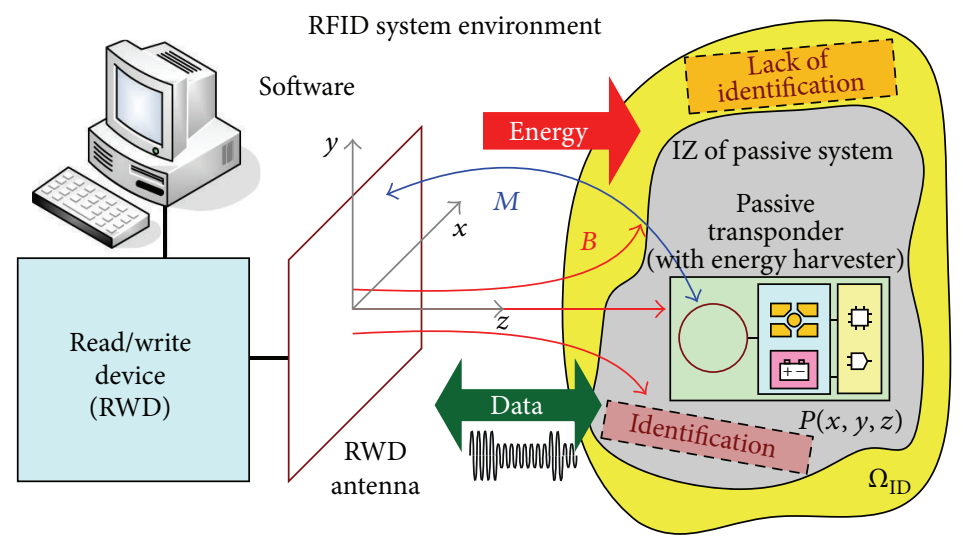

FIGURE 2: Passive RFID system with inductive coupling and energy harvesting mechanism.

of low power integrated circuits as well as trends towards utilizing alternative energy that can be harvested from the operating environment of RFID systems are the reasons why many current investigations are focused on the integration of sensors with passive transponders [25-29]. The research results are useful in many applications of contactless automatic identification (medicine [30] and healthcare [31, 32], building industry [33], and supply chains [34]), although it is impossible to save measurement data in memory of such a hybrid construction without activation of RWD devices.

On the base of elaborated 3D IZ model, authors present (Section 2) the aspects connected with recovering excess energy which is supplied by an RWD device and with its utilization to power a pressure sensor with a passive RFID chip. Using the model and a passive chip with energy harvester, they propose the construction of sensor with RFID interface (Section 3) that is designed to work in the HF band of inductively coupled RFID systems (operating frequency $f_{0}=$ $13.56 \mathrm{MHz}$ ), according to the communication protocol ISO/IEC 15693 [35]. Analyzing conducted tests and derived results (Section 4), readers can see that synthesis model of $3 \mathrm{D}$ interrogation zone can be used for predicting spatial placements of sensors in different kinds of RFID system applications [36].

\section{Model of Passive Transponder with Energy Harvester}

RFID systems working in the HF band utilize the typical operating frequency $f_{0}=13.56 \mathrm{MHz}$. Because the wavelength $\lambda$ is about $22 \mathrm{~m}$, the RWD and transponder antennas are made in the form of small loop in relation to $\lambda$. The inhomogeneous magnetic field generated into the RWD antenna vicinity is the medium for both transferring energy and wireless data exchange. The most common mean of data transmission is the load modulation with amplitude-shift keying. The load modulation with subcarrier is used because of the necessity for transferring energy to the passive transponders. These mechanisms are implemented in protocols normalized by ISO/IEC 15693, 14443, 18000-3, and others.

The inductively coupled RFID HF systems operate in the zone for which an inhomogeneous magnetic field (characterised by the induction $B$ or magnetic field strength $H$ ) and strong coupling (characterised by the mutual inductance $M$ ) between antennas of the communication set occur (Figure 2).

The efficiency estimation of energy transmission from RWD to passive transponder is complicated for this kind of medium, especially in the proposed solution with autonomous features (e.g., module for measuring physical quantities). Since the extra module disturbs the proper operation of transponder, the careful study of its impact on main parameters is compulsory. This problem is explained in details on the elaborated model of a passive transponder with the active build-in block for harvesting energy from the RFID system environment (Figure 3).

The presented model is valid for all antenna arrangements existing in proximity [37] or long range [38] RWD devices. It is suitable for the transponder located in a $P(x, y, z)$ point of the Cartesian coordinate system (Figure 2). It includes all elements of real solution: loop antenna, chip with extra energy harvester, and in addition microprocessor for controlling autonomous features. The microprocessor system tasks are to gather data from sensors (e.g., by using $\mathrm{A} / \mathrm{C}$ converter) and write it to a chip internal memory by using a communication interface (e.g., $\mathrm{I}^{2} \mathrm{C}$ bus). It is powered by the harvester and it is active only when the transponder is in the interrogation zone and the energy conditions are satisfied.

The antenna loop is a parallel circuit in which $L_{T}$ is the self-inductance and $R_{T}$ represents the resistance of wire used for creating the winding and it also characterizes ohmic losses. The electric capacity $C_{T S}$ is the resultant of all capacitances between the coils and it results from a uniform distribution of wire electrical parameters along the entire length of winding. The equivalent of loop antenna has been also included in the general scheme in order to facilitate a subsequent experimental verification $\left(R_{T S}\right.$ and $L_{T S}$ denote the serial resistance and the inductance of series antenna circuit). The source $U_{R T}$ represents voltage inducing in the antenna loop when the transponder is in the magnetic field of read/ write device. It is expressed by (1) where pulsation $\omega=2 \pi f_{0}$ and $I_{R}$ means the current in the winding as

$$
U_{R T}=j \omega \cdot M \cdot I_{R}
$$




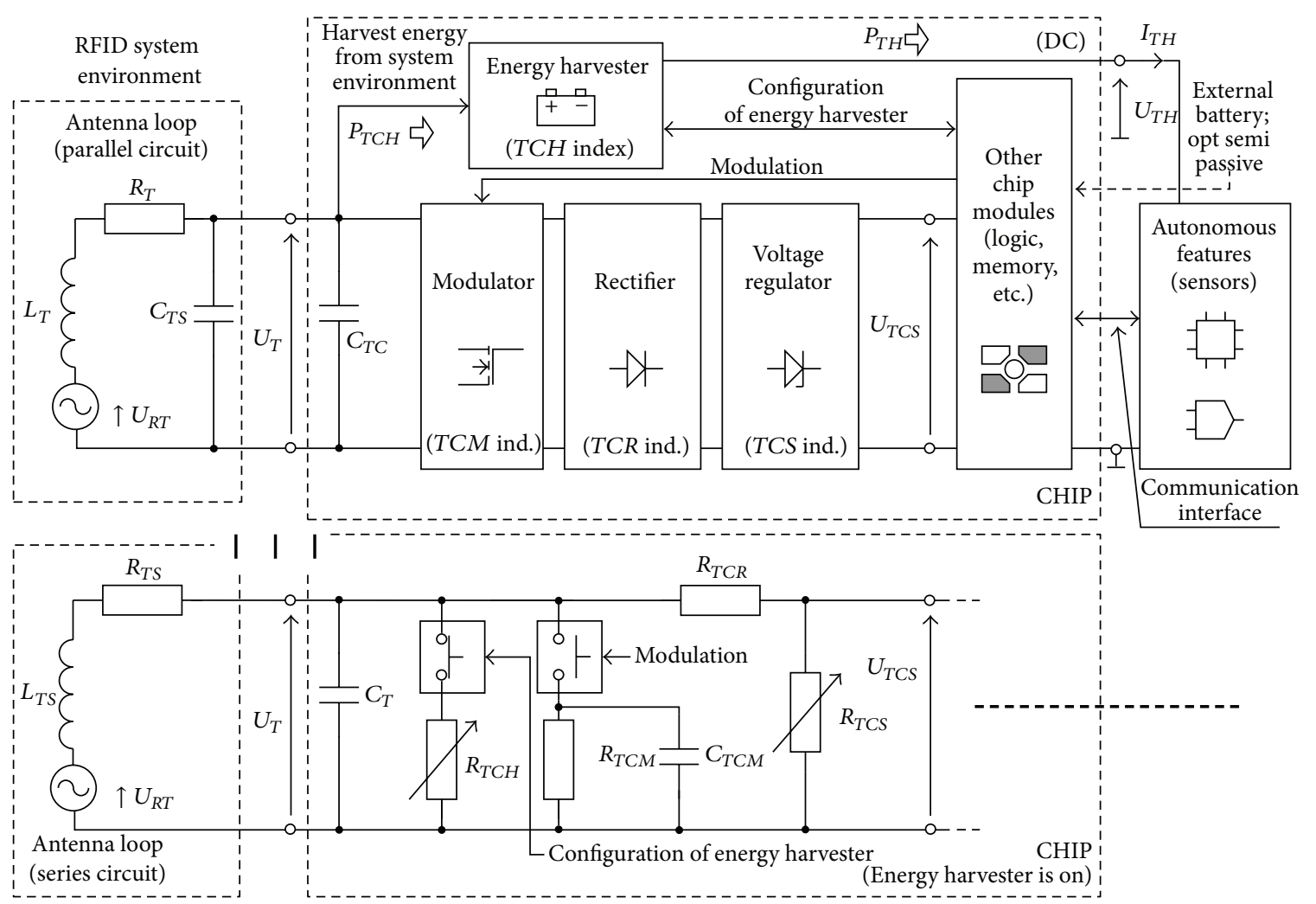

FIGURE 3: Model and electric equivalent of passive RFID transponder with energy harvester.

The maximum value of voltage $U_{T}$ on loop antenna terminals is obtained for the parallel resonance between the inductance $L_{T}$ and the capacitance $C_{T}$ of active chip. The capacitance $C_{T}$ is expressed by (2) where $C_{T C}$ means the selfcapacity of chip (an energy storage cell for powering radio communication processes), $C_{T C R}$ is the resultant of rectifier and voltage regulator capacitances, and $C_{T C H}$ describes the electric capacity of energy harvester as

$$
C_{T}=C_{T C}+C_{T C R}+C_{T C H} \cong C_{T C} \text {. }
$$

The synthesis of rectifier and voltage regulator is made separately $[39,40]$. Although the rectification takes place in a half-wave or full-wave rectifier [41], it is controlled by the voltage regulator [39]. In the case of passive transponders working in the HF band, both of the circuits are commonly realized on fast switching diodes in MOS technology [42]. It should be mentioned that the proposed model will be also valid for all blocks manufactured on a flexible substrate in a printed electronic technology which is possible thanks to the tremendous progress in the nanomaterial science $[43,44]$.

The rectifier and voltage regulator are characterized by the capacitances included in the $C_{T}$ quantity and by the resistances $R_{T C R}$ and $R_{T C S}$. These elements of transponder equivalent affect electric circuit parameters which are seen at the loop antenna terminals. The $R_{T C R}$ is a generalized parameter of voltage rectifier circuit obtained on the base of diode graphical model (static characteristic). Its value mainly depends on a circuit design and elements included in a specified construction of chip from a given manufacturer. Since the piecewise linear approximation of diode characteristic is generally used in electronic circuit analyses, the linear nature can be assumed for this value. The second quantity $R_{T C S}$ represents the internal structure of voltage regulator. Although this block is used for powering the internal structure of transponders, it can be assumed that the stabilized voltage $U_{T C S}$ is constant. This approximation is justified due to negligibly small current drawn and limited impact of this phenomenon on circuit parameters seen at the loop antenna terminals.

The variable resistance $R_{T C H}$ represents the harvester internal structure. If minimum losses are assumed in this block (the power $P_{T C H}$ at the input is almost equal to the power $P_{T H}$ supplied to the autonomous feature module), the resistance value can be determined from (3) in which $U_{T R M S}$ means RMS voltage $U_{T}$ and $U_{T H}, I_{T H}$, respectively, dc current and voltage on the power output of energy harvesting block as

$$
R_{T C H}=\frac{U_{T R M S}^{2}}{P_{T C H}} \cong \frac{U_{T H}}{I_{T H}} .
$$

Since the harvester derives energy from the electromagnetic field of read/write device and therefore affects the interrogation zone, the possibility of its functional property configuration is usually available by manufacturers. Depending on a chip design, it is possible to reduce or control the output values of voltage $U_{T H}$ and/or maximum current $I_{T H}[45,46]$. These properties describe appropriate operation boundaries 
of energy recovery block and ability to power the autonomous function modules together with connected sensors.

The modulator capacitance $C_{T C M}$ and resistance $R_{T C M}$ are also included in the model (Figure 3). The proper transponder operation does not depend on these parameters in the considered range. It is due to the fact that radio-communication processes are conducted according to adequate protocols (for the HF band: ISO/IEC 15693, 14443, 18000-3, and others) and the load modulation with subcarrier is used for transmitting data in the transponder-RWD direction.

Taking into account the underlying assumptions, it is possible to determine the voltage value induced at the loop antenna terminals under the load of modelled chip input circuits (4). Then, the voltage drops in the unloaded divider $R_{T C R}$ and $R_{T C S}$ are described by (5) as

$$
\begin{gathered}
U_{T}=j \omega \cdot M \cdot I_{R} \\
\times\left(1+\left(\left(1 / R_{T C H}\right)+\left(1 /\left(R_{T C R}+R_{T C S}\right)\right)+j \omega C_{T}\right)\right. \\
\left.\times\left(j \omega L_{T S}+R_{T S}\right)\right)^{-1} \\
U_{T}=U_{T C S} \cdot\left(1+\frac{R_{T C R}}{R_{T C S}}\right) .
\end{gathered}
$$

Because the $U_{T C S}$ part is constant for $U_{T}>U_{T C S}$, the variable resistance $R_{T C S}$ of voltage regulator is given by (6)

$$
\begin{gathered}
R_{T C S}=\mid U_{T C S}\left(R _ { T C H } \left(\omega^{2} L_{T S} C_{T} R_{T C R}-R_{T C R}-j \omega L_{T S}\right.\right. \\
\left.-j \omega C_{T} R_{T S} R_{T C R}-R_{T S}\right) \\
\left.-R_{T S} R_{T C R}-j \omega L_{T S} R_{T C R}\right) \\
\times\left(U _ { T C S } \left(R_{T C H}\left(1+j \omega C_{T} R_{T S}-\omega^{2} L_{T S} C_{T S}\right)\right.\right. \\
\left.\left.+R_{T S}+j \omega L_{T S}\right)-j \omega M I_{R} R_{T C H}\right)^{-1} \mid .
\end{gathered}
$$

The minimum value of $U_{T}\left(U_{T \text { min }}\right)$ voltage is the base for determining the interrogation zone. The $U_{T \text { min }}$ value is the characteristic parameter of chip construction. It clearly impacts on (7) (where $\mu_{0}=4 \pi \cdot 10^{-7} \mathrm{H} / \mathrm{m}$ ) and on its base it is possible to determine the minimum value of magnetic field strength $H_{\min }$ as

$$
\begin{aligned}
H_{\min }= & \frac{\left|U_{T \min }\right|}{\mu_{0}} \\
& \cdot \mid\left[1+\left(\left(1 / R_{T C H}\right)+\left(1 /\left(R_{T C R}+R_{T C S}\right)\right)+j \omega C_{T}\right)\right. \\
& \left.\cdot\left(j \omega L_{T S}+R_{T S}\right)\right]\left(j \omega \cdot N_{T} \cdot S_{T}\right)^{-1} \mid .
\end{aligned}
$$

The field strength $H_{\min }$ is the elementary parameter that defines the IZ. It is differentiated on the base of value $U_{T \text { min }}$ for the given direction of data transmission and the kind

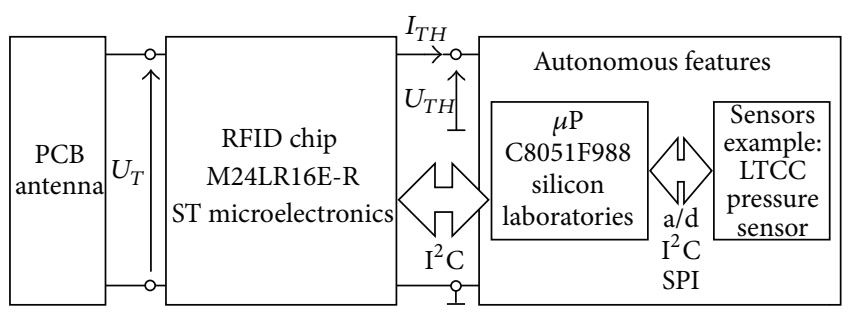

FIgURE 4: Block diagram of the elaborated transponder.

of operations (read/write) proceeded in the internal transponder's memory. The operation of transponderwith the active harvester is also described by (7). If the $1 / R_{\mathrm{TCH}}$ factor is omitted then the $H_{\text {min }}$ parameter is valid for the typical passive transponder.

\section{Implementation of Passive Chip with Energy Harvester}

The special laboratory stand has been made for experimental verification of the calculated value $H_{\min }$. The stand allows to measure the maximum distance between the RWD and transponder antennas for which the correct operation of RFID system is ensured.

The batteryless system has been made in order to exemplify the possibility of passive chip integration with the previously presented pressure sensor [9]. The special circuit for recovering energy from the RFID system environment is the built-in chip (Figure 4). The presented solution is dedicated for transponders that work in inductively coupled RFID systems in the HF band $\left(f_{0}=13.56 \mathrm{MHz}\right)$ and operate according to the communication protocol ISO/IEC 15693 [35].

The chip M24LR16E-R STMicroelectronics [45] is an integral part of transponder. It is equipped with a $16 \mathrm{~kb}$ EEPROM with a password protection. The memory organisation depends on the access mode: 2048 B in $\mathrm{I}^{2} \mathrm{C}$ mode and 512 blocks of $32 \mathrm{~b}$ in RF mode. The access is possible by a dual interface: wireless RFID and serial wire link $\mathrm{I}^{2} \mathrm{C}$. The energy harvesting block can operate in four configurable ranges of current sink-the maximum value of $I_{T H \max }$ (Figure 3 ) is equal to $6 \mathrm{~mA}, 3 \mathrm{~mA}, 1 \mathrm{~mA}$, or $300 \mu \mathrm{A}$. The internal tuning capacitance $\left(C_{T}=27.5 \mathrm{pF}\right.$ for $\left.1 \mathrm{~V}_{\mathrm{pp}}\right)$ is included at the loop antenna terminals.

The square antenna has been synthesised in order to carry out the experimental verification. The winding diagram of loop antenna (Figure 5(a)) has been developed in the HyperLynx 3D EM 15.21 package (Mentor Graphics). The test antenna has been realized practically on a PCB substrate by using a CNC plotter LPKF ProtoMat S100 (Figure 5(b)).

The project has been prepared for the typical twosided FR-4 laminate (thickness: $1.55 \mathrm{~mm}$, permittivity: 4.85 , dielectric losses: 0.025 for $f=10 \mathrm{MHz}$, thickness of copper: $17.5 \mu \mathrm{m})$ and for the assumed parameters of applied chip $\left(C_{T}=27.5 \mathrm{pF}, f_{0}=13.56 \mathrm{MHz}\right)$. The loop parameters of model calculated in the HyperLynx 3D EM package have been confirmed experimentally by measuring the test 


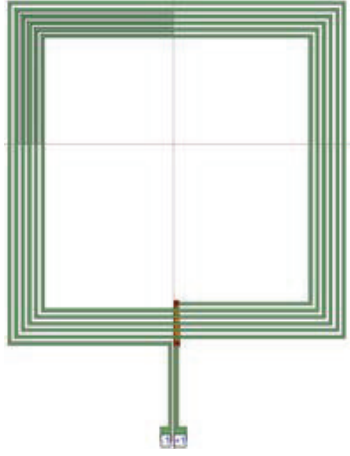

(a)

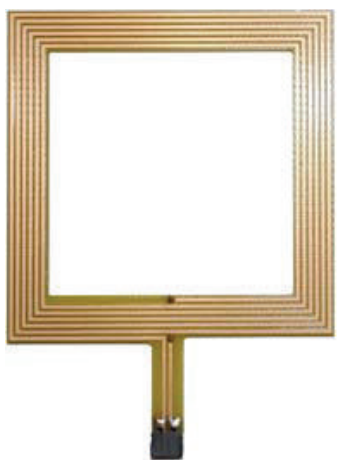

(b)

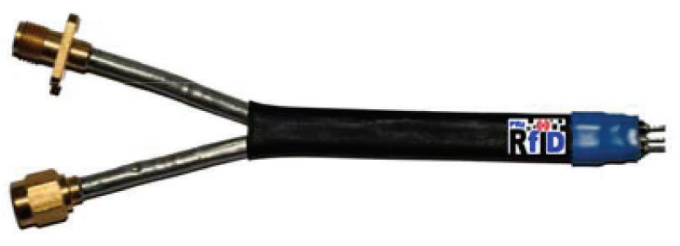

(c)

Figure 5: Transponder antenna: (a) HyperLynx 3D EM winding model, (b) test antenna, and (c) differential probe.

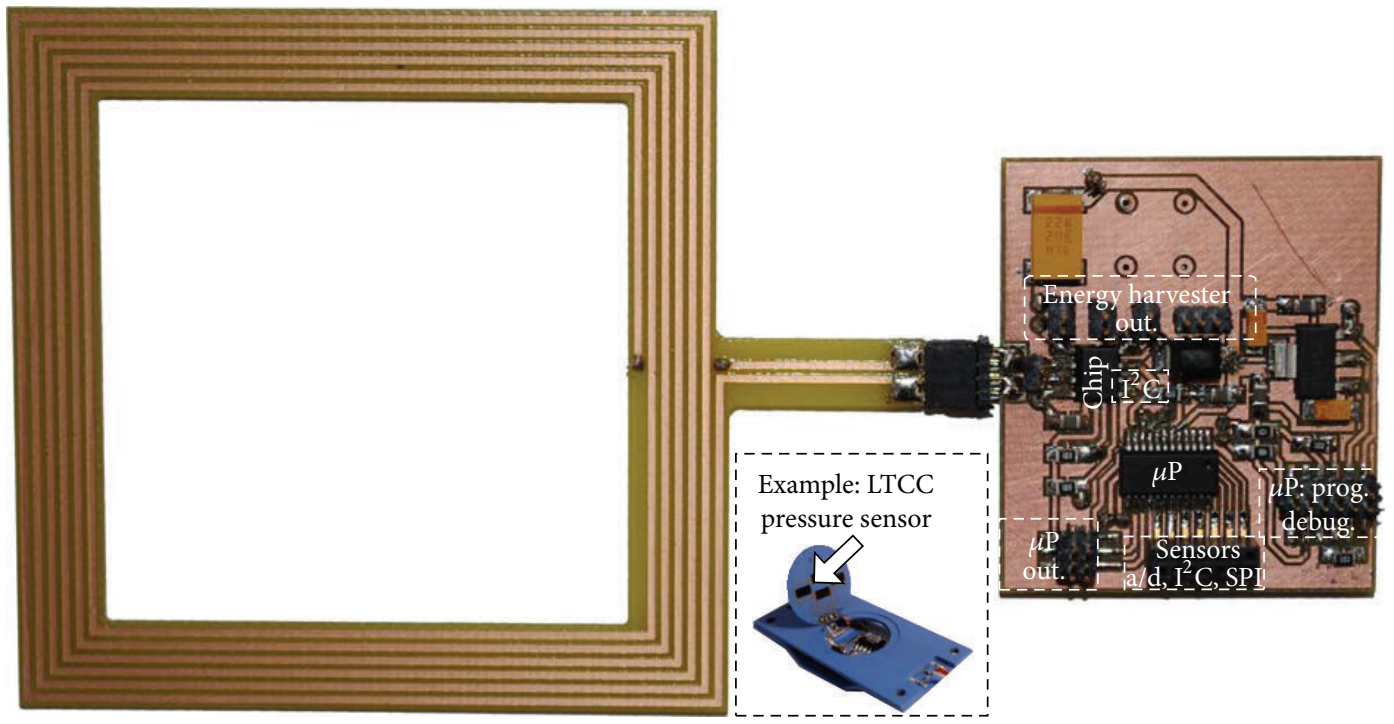

FIgURE 6: Practical realization of the transponder with harvester of additional energy.

antenna using a two-port network analyzer (Agilent PNA-X $\mathrm{N} 5242 \mathrm{~A}$ ) and the differential probe (Figure 5(c)) which has been prepared especially for connecting equipment without any disturbances.

The antenna impedance $Z_{T}$ has been calculated from (8) on the basis of measured $S$-parameters [47] (for $Z_{0}=50 \Omega$ ) as

$$
Z_{T}=j \omega L_{T S}+R_{T S}=2 Z_{0} \frac{\left(1-S_{11}^{2}+S_{21}^{2}-2 S_{12}\right)}{\left(1-S_{11}\right)^{2}-S_{21}^{2}} .
$$

The convergence of measured and calculated data confirms the accuracy of realized project (Table 1). The parallel resonance with the $C_{T}$ capacitance at $f_{0}$ frequency is achievable for obtained parameters. It means that this test antenna construction allows to effectively transfer energy from the RWD to the chip and additionally to power the extra feature blocks.

The expanded sensor block is built on the C8051F988 microprocessor (Figure 6). This (integrated circuit) IC is the industry's lowest power microcontroller of 8051 family (MCU). It consumes as little as $10 \mathrm{nA}$ in a sleep mode (with
TABLE 1: Calculated and measured parameters of the transponder antenna.

\begin{tabular}{lcc}
\hline Parameter & HyperLynx calculation results & Measurement results \\
\hline LTS & $5.08 \mu \mathrm{H}$ & $5.10 \mu \mathrm{H}$ \\
RTS & $8.59 \Omega$ & $8.68 \Omega$ \\
\hline
\end{tabular}

full memory retention) and $150 \mu \mathrm{A} / \mathrm{MHz}$ in an active mode, which saves power when the application runs. It is capable of operating down to $1.8 \mathrm{~V}$. It also offers the industry's fastest wake-up and analog settling time. It is equipped with all functions needed in the project: 10-bit $\mathrm{AD}$ converter for connecting the LTCC sensor, $\mathrm{I}^{2} \mathrm{C}$ interface for transferring data to the RFID chip, and 2 built-in supply monitors (brown-out detector) for the sleep and active modes.

\section{Tests and Results}

Designed elements of HF long range RFID systems with inductive coupling (working frequency: $f_{0}=13.56 \mathrm{MHz}$ ) 


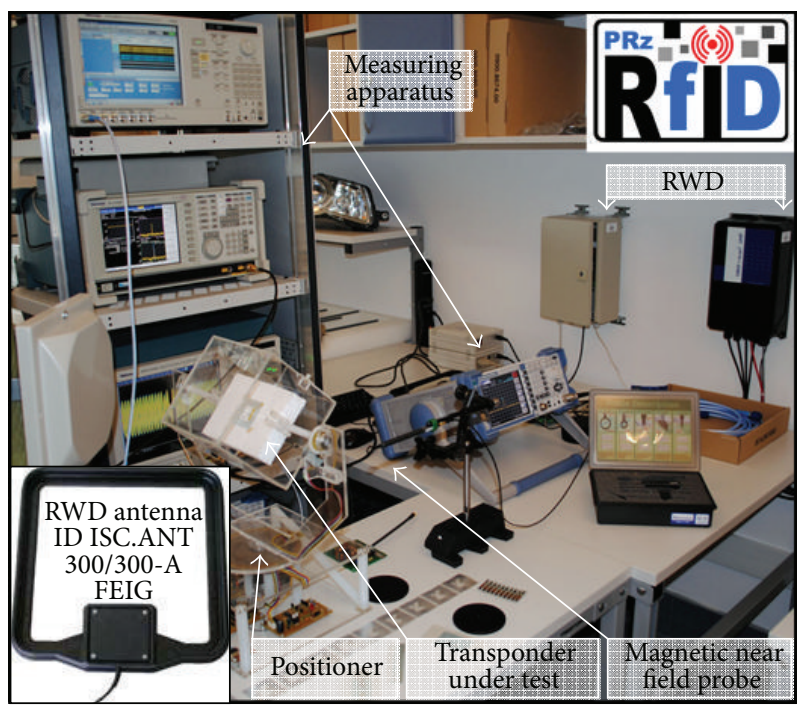

Figure 7: Test stand in the RFID laboratory in the Department of Electronic and Communications Systems.

have been used to carry out final tests of the elaborated method useful for determining the minimum value of magnetic field strength $H_{\min }$ (read range). All measurements of the experiment have been performed in the specialized RFID laboratory. The special test stand has been prepared for this reason (Figure 7). The parameters used in the equivalent calculations have corresponded to the parameters of devices available in the laboratory. The laboratory equipment has made it possible to conduct all kinds of experimental research in the range of inductively coupled RFID system activity, in both the energy and communication aspects.

The square RWD antenna (side length $a=0.3 \mathrm{~m}$, number of loop turns $N_{R}=1$ ) has been installed in the test stand. The antenna was supplied by the RWD ID ISC.LR200 FEIG (output power $1 \mathrm{~W}$, current in the winding $I_{R}=0.45 \mathrm{~A}$ ). The calculation and measurement results of magnetic field strength in the symmetry axis of RWD antenna are presented in Figure 8.

The calculation has been made for the antenna model that had been considered in [38] in details. The calculated results have been compared with the measurements obtained in the laboratory stand using spectrum analyzer R\&S FSL18, oscilloscope Tektronix DPO71254B, magnetic near-field probe HZ14, and also P7504, CT1.

On the base of calculated and measured result convergence, the correctness of magnetic field strength determination in the elaborated test stand has been confirmed. The possibility of energy harvesting on different kinds of its value level has been also estimated. It has been necessary in order to establish appropriate conditions for powering the autonomous functions of the designed transponder. The measurement results of chip harvester efficiency are presented in Table 2. The measurements have been made by a Tektronix DMM4020 digital multimeter. The power consumption variations have been obtained by using elaborated load simulator

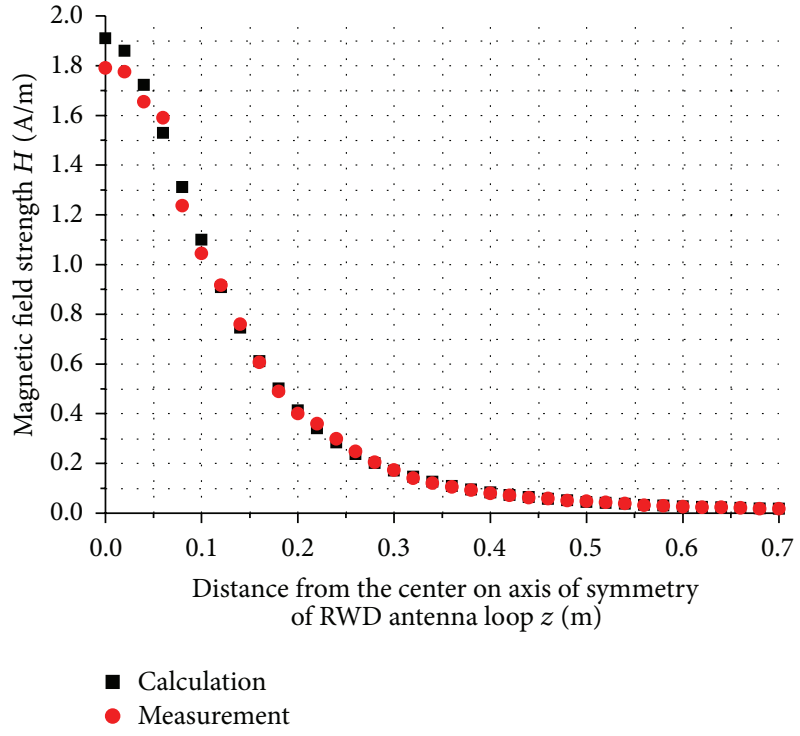

FIGURE 8: Magnetic field strength in test stand.

TABle 2: Measurements of power efficiency for energy harvesting block.

\begin{tabular}{lccc}
\hline $\begin{array}{l}\text { RFID chip configuration } \\
\left.\text { (maximum current } I_{T H}\right)\end{array}$ & $I_{T H}$ & $U_{T H}$ & $P_{T H}$ \\
$\mathrm{~mA}$ & $\mathrm{~mA}$ & $\mathrm{~V}$ & $\mathrm{~mW}$ \\
\hline \multirow{6}{*}{3} & 0.30 & 3.28 & 1.0 \\
& 0.50 & 3.20 & 1.6 \\
& 0.70 & 3.13 & 2.2 \\
& 0.90 & 3.05 & 2.7 \\
& 1.00 & 3.02 & 3.0 \\
& 1.20 & 2.95 & 3.5 \\
& 1.40 & 2.89 & 4.0 \\
& 1.60 & 2.83 & 4.5 \\
& 1.80 & 2.77 & 5.0 \\
& 2.00 & 2.72 & 5.4 \\
& 3.00 & 2.51 & 7.5 \\
\hline \multirow{3}{*}{6} & 4.00 & 2.30 & 9.2 \\
& 5.00 & 2.11 & 10.6 \\
\hline
\end{tabular}

(it changed the power levels according to predicted activities of microprocessor control system).

The main application parameters of test transponders have been determined on the base of above mentioned calculations and measurements (Table 3 ).

The calculations have been performed for transponder model described by (6) and (7). The mathcad environment has been used for preparing calculation program tools. The data in Table 3 represents three modes of designed transponder: (1) energy harvester OFF, (2) energy harvester $\mathrm{ON}$ and $P_{T H}=1 \mathrm{~mW}$, and (3) energy harvester $\mathrm{ON}$ and $P_{T H}=$ $4 \mathrm{~mW}$. The power $P_{T H}$ has been measured by an oscilloscope Tektronix DPO71254B with probes P7504 CT1 (index ${ }^{a}$ in Table 3). 
TABLE 3: Calculated and measured parameters of the developed transponder.

\begin{tabular}{|c|c|c|c|c|c|}
\hline Transponder state & Status of energy harvester & $P_{T H}$ & Parameter & Calculating results & Measuring results \\
\hline \multirow{2}{*}{1} & \multirow{2}{*}{ OFF } & \multirow{2}{*}{${ }^{\mathrm{a}} 0 \mathrm{~mW}$} & $z_{I D}$ & - & ${ }^{\mathrm{b}} 0.6 \mathrm{~m}$ \\
\hline & & & $H_{\min }$ & $\begin{array}{l}{ }^{\mathrm{c}} 0.028 \mathrm{~A} / \mathrm{m} \\
{ }^{\mathrm{d}} 0.027 \mathrm{~A} / \mathrm{m}\end{array}$ & ${ }^{\mathrm{e}} 0.027 \mathrm{~A} / \mathrm{m}$ \\
\hline \multirow[t]{2}{*}{2} & \multirow{2}{*}{$\mathrm{ON}$} & \multirow{2}{*}{${ }^{\mathrm{a}} 1 \mathrm{~mW}$} & $z_{I D}$ & - & ${ }^{\mathrm{b}} 0.35 \mathrm{~m}$ \\
\hline & & & $H_{\min }$ & $\begin{array}{l}{ }^{\mathrm{c}} 0.120 \mathrm{~A} / \mathrm{m} \\
{ }^{\mathrm{d}} 0.121 \mathrm{~A} / \mathrm{m}\end{array}$ & ${ }^{\mathrm{e}} 0.123 \mathrm{~A} / \mathrm{m}$ \\
\hline \multirow{2}{*}{3} & \multirow{2}{*}{ ON } & \multirow{2}{*}{${ }^{\mathrm{a}} 4 \mathrm{~mW}$} & $z_{I D}$ & - & ${ }^{\mathrm{b}} 0.19 \mathrm{~m}$ \\
\hline & & & $H_{\min }$ & $\begin{array}{l}{ }^{\mathrm{c}} 0.461 \mathrm{~A} / \mathrm{m} \\
{ }^{\mathrm{d}} 0.454 \mathrm{~A} / \mathrm{m}\end{array}$ & ${ }^{\mathrm{e}} 0.462 \mathrm{~A} / \mathrm{m}$ \\
\hline
\end{tabular}

${ }^{\mathrm{a}}$ Measurement: oscilloscope Tektronix DPO71254B, probes P7504, CT1.

${ }^{\mathrm{b}}$ UID measurement: inventory command (0x01) ISO/IEC 15693 (FEIG ID ISOStart 2011 version 08.03.01).

${ }^{c}$ Calculation: equation (7) for RWD-transponder model (square RWD antenna model [38]; transponder antenna loop: HyperLynx 3D EM model; chip: product specification data).

${ }^{\mathrm{d}}$ Calculation: RWD antenna model on the base of measured $z_{I D}$.

${ }^{\mathrm{e}}$ Measurement: spectrum analyzer R\&S FSL18, magnetic near-field probe HZ-14.

The real communication process has been carried out between the RWD device and the prepared test samples of analyzed transponder and the transmission correctness has been controlled by the spectrum analyzer Tektronix RSA 3408B. The parameter $z_{I D}$ has been determined as the basic quantity of the interrogation zone and it denotes the maximum operating distance (read range) of transponder located in the symmetry axis of RWD antenna loop. The index ${ }^{b}$ in Table 3 means the measurement of unique identifier (UID) in the test stand for inventory command (0x01) ISO/IEC 15693 (FEIG ID ISOStart 2011 version 08.03.01).

Calculations of the $H_{\min }$ have been made by two methods. The index ${ }^{c}$ in Table 3 means that the calculations result from (7) for the RWD-transponder model (square RWD antenna model [38]; transponder antenna loop: HyperLynx 3D EM model; chip: product specification data). The index ${ }^{\mathrm{d}}$ in Table 3 means that the calculations result from RWD antenna model derived on the base of measured $z_{I D}$. The minimum value of magnetic field strength $H_{\min }$ (index ${ }^{\mathrm{e}}$ in Table 3) has been measured by means of the spectrum analyzer R\&S FSL18 and magnetic near-field probe HZ-14.

The result convergence confirms the accuracy and correctness of developed simulation equivalent to real passive transponder with harvesting module.

\section{Conclusion}

The basic application parameters presented in Table 3 have been calculated for the passive transponder electric circuit equivalent and also the same quantities have been measured for the test electronic circuit. The verification of the developed model is confirmed on the base of the obtained result convergence. The developed methodology for testing passive and semipassive transponders with harvester that derives energy from RFID system environment is the key approach to determine the tree-dimensional interrogation zone (e.g., by using Monte Carlo method [36]) for both single and anticollision RFID systems. It provides reliable information on the operation and efficiency of automated identification processes and it allows RFID system designers to eliminate inefficient and time-consuming trial and error methods.

\section{Acknowledgments}

This work was supported in part by the Polish National Science Centre (NCN) under Grant no. 4711/B/T02/2011/40 (in the scope of RFID sensor) and the Polish National Centre for Research and Development (NCBR) under Grant no. PBS1/A3/3/2012 (in the scope of energy harvesting in RFID transponders). This work was developed by using equipment purchased in the Operational Program Development of Eastern Poland 2007-2013 of the Priority Axis I Modern Economics of Activity I.3 Supporting Innovation under Grant no. POPW.01.03.00-18-012/09-00 and the Program of Development of Podkarpacie Province of The European Regional Development Fund under Grant no. UDA-RPPK.01.03.00-18003/10-00.

\section{References}

[1] D. Brown, "Part II: applications," in RFID Implementation, pp. 133-222, McGraw-Hill, New York, NY, USA, 2007.

[2] E. C. Jones and C. A. Chung, "Part 2: integrating RFID into logistics," in RFID in Logistics: A Practical introduction, CRC Press, New York, NY, USA, 2008.

[3] K. Weigelt, M. Hambsch, G. Karacs, T. Zillger, and A. C. Hübler, "Labeling the world: tagging mass products with printing processes," IEEE Pervasive Computing, vol. 9, no. 2, pp. 59-63, 2010.

[4] S. Cheng, K. Tom, L. Thomas, and M. Pecht, "A wireless sensor system for prognostics and health management," IEEE Sensors Journal, vol. 10, no. 4, pp. 856-862, 2010.

[5] V. P. Plessky and L. M. Reindl, "Review on SAW RFID tags," IEEE Transactions on Ultrasonics, Ferroelectrics, and Frequency Control, vol. 57, no. 3, pp. 654-668, 2010.

[6] R. Bridelall and A. Hande, "Novel RFID technologies: energy harvesting for self-powered autonomous RFID systems," in RFID Systems: Research Trends and Challenges, M. Bolić, D. 
Simplot-Ryl, and I. Stojmenović, Eds., pp. 473-495, Wiley, New York, NY, USA, 2010.

[7] V. Pillai, H. Heinrich, D. Dieska, P. V. Nikitin, R. Martinez, and K. V. S. Rao, "An ultra-low-power long range battery/passive RFID tag for UHF and microwave bands with a current consumption of $700 \mathrm{nA}$ at 1.5 V,' IEEE Transactions on Circuits and Systems I, vol. 54, no. 7, pp. 1500-1512, 2007.

[8] N. D. Phan, I. J. Jang, and J. W. Lee, "A 2-Kb one-time programmable memory for UHF passive RFID tag IC in a standard 0. $18 \mu \mathrm{m}$ CMOS process," IEEE Transactions on Circuits and Systems I, no. 99, pp. 1-13, 2012.

[9] S. Slosarczyk, I. Vehec, W. Kalita, R. Bauer, and W. Sabat, "3Dtvarovany modul s integrovanym senzorom tlaku," The AAPS Journal, pp. 228-230, 2007.

[10] K. Finkenzeller, "Differentiation features of RFID systems," in RFID Handbook: Fundamentals and Applications in Contactless Smart Cards, Radio Frequency Identification and Near-Field Communication, pp. 11-28, Wiley, New York, NY, USA, 3rd edition, 2010.

[11] P. Jankowski-Mihułowicz and W. Kalita, "Interrogation zonea basic application parameter of RFID systems," Electronics, no. 8, pp. 67-72, 2010.

[12] P. Jankowski-Mihułowicz, W. Kalita, and B. Pawlowicz, "Problem of dynamic change of tags location in anticollision RFID systems," Microelectronics Reliability, vol. 48, no. 6, pp. 911-918, 2008.

[13] C. W. Lee, S. J. Lee, M. Kim, Y. Kyung, and K. Eom, “Capacitive humidity sensor tag smart refrigerator system using the capacitive to voltage converter (CVC)," in International Journal of Science and Advanced Technology, pp. 15-26, 2011.

[14] E. Abad, B. Mazzolai, A. Juarros et al., "Fabrication process for a flexible tag microlab," in Smart Sensors, Actuators, and MEMS III, vol. 6589 of Proceedings of SPIE, p. 5890, Gran Canaria, Spain, May 2007.

[15] A. Oprea, N. Bârsan, U. Weimar, M. Bauersfeld, D. Ebling, and J. Wöllenstein, "Capacitive humidity sensors on flexible RFID labels," Sensors and Actuators B, vol. 132, no. 2, pp. 404-410, 2008.

[16] T. Volk, D. Jansen, H. Speletz, B. Fleiner, D. Bau, A. Kreker et al., "Active RFID sensor with integrated file system for logistic applications," in Proceedings of the European Workshop on Smart Objects: Systems, Technologies and Applications, pp. 1-7, June 2010.

[17] S. Kim, J. Cho, H. Kim, H. Kim, H. Kang, and S. Hong, "An EPC Gen 2 compatible passive/semi-active UHF RFID transponder with embedded FeRAM and temperature sensor," in Proceedings of the IEEE Asian Solid-State Circuits Conference (A-SSCC '07), pp. 135-138, November 2007.

[18] D. Cartasegna, A. Cito, F. Conso, A. Donida, M. Grassi, L. Malvasi et al., "Smart RFID label for monitoring the preservation conditions of food," in Sensors and Microsystems, vol. 54 of Lecture Notes in Electrical Engineering, pp. 381-385, Springer, Berlin, Germany, 2010.

[19] A. Oprea, J. Courbat, N. Bârsan, D. Briand, N. F. de Rooij, and U. Weimar, "Temperature, humidity and gas sensors integrated on plastic foil for low power applications," Sensors and Actuators B, vol. 140, no. 1, pp. 227-232, 2009.

[20] L. Yang, A. Rida, T. Wu, S. Basat, and M. M. Tentzeris, "Integration of sensors and inkjet-printed RFID tags on paperbased substrates for UHF cognitive intelligence applications," in Proceedings of the IEEE Antennas and Propagation Society
International Symposium (AP-S '07), vol. 1-12, pp. 1193-1186, June 2007.

[21] A. Tani, M. Ugaji, and Y. Yamabe, "A building structuralperformance monitoring system using RFID tag with sensors," in Proceedings of the International Conference on Computing in Civil Building Engineering, p. 441, 2010.

[22] E. Abad, S. Zampolli, S. Marco et al., "Flexible tag microlab development: gas sensors integration in RFID flexible tags for food logistic," Sensors and Actuators B, vol. 127, no. 1, pp. 2-7, 2007.

[23] ERC, Recommendation 70-03 Relating to the Use of Short Range Devices (SRD), Version of 22 August, ERC, New York, NY, USA, 2011.

[24] S. Mandal and R. Sarpeshkar, "Low-power CMOS rectifier design for RFID applications," IEEE Transactions on Circuits and Systems I, vol. 54, no. 6, pp. 1177-1188, 2007.

[25] Y. Jia, M. Heiß, Q. Fu, and N. A. Gay, "A prototype RFID humidity sensor for built environment monitoring," in Proceedings of the 2008 International Workshop on Education Technology and Training and 2008 International Workshop on Geoscience and Remote Sensing, vol. 2, pp. 496-499, December 2008.

[26] J. Virtanen, L. Ukkonen, T. Björninen, A. Z. Elsherbeni, and L. Sydänheimo, "Inkjet-printed humidity sensor for passive UHF RFID systems," IEEE Transactions on Instrumentation and Measurement, vol. 60, no. 8, pp. 2768-2777, 2011.

[27] A. Vazt, H. Solart, I. Rebollot, I. Gutierrezt, and R. Berenguert, "Long range, low power UHF RFID analog front-end suitable for battery less wireless sensors," in Proceedings of the IEEE MTT-S International Microwave Symposium Digest (MTT '10), pp. 836-839, May 2010.

[28] I. Fernandez, A. Asensio, I. Gutierrez, J. Garcia, I. Rebollo, and J. de No, "Study of the communication distance of a MEMS pressure sensor Integrated in a RFID passive tag," Advances in Electrical and Computer Engineering., vol. 12, no. 1, pp. 15-18, 2012.

[29] Z. Zou, D. S. Mendoza, P. Wang et al., "A low-power and flexible energy detection IR-UWB receiver for RFID and wireless sensor networks," IEEE Transactions on Circuits and Systems I, vol. 58, no. 7, pp. 1470-1482, 2011.

[30] M. Ghovanloo and S. Atluri, "An integrated full-wave CMOS rectifier with built-in back telemetry for RFID and implantable biomedical applications," IEEE Transactions on Circuits and Systems I, vol. 55, no. 10, pp. 3328-3334, 2008.

[31] E. Y. Chow, A. L. Chlebowski, S. Chakraborty, W. J. Chappell, and P. P. Irazoqui, "Fully wireless implantable cardiovascular pressure monitor integrated with a medical stent," IEEE Transactions on Biomedical Engineering, vol. 57, no. 6, pp. 1487-1496, 2010.

[32] Y. Shih, T. Shen, and B. P. Otis, "A $2.3 \mu \mathrm{W}$ wireless intraocular pressure/temperature monitor," IEEE Journal of Solid-State Circuits, vol. 46, no. 11, pp. 2592-2601, 2011.

[33] M. van Ackeren and M. Bollerott, Humidity and Temperature Transponder, Fraunhofer Institute for Microelectronic Circuits and Systems, Duisburg, Germany, 2010.

[34] EU-Commision, Information and Society-and-Media, Internet of Things in 2020-Roadmap For the Future, INFSO D. 4 Networked Enterprise \& RFID, INFSO G. 2 Micro \& Nanosystems, Working Group RFID of The ETP EPoSS, 2008.

[35] ISO, "Identification cards-contactless integrated circuit(s) cards-vicinity cards-part 3: anticollision and transmission protocol," ISO/IEC 15693-3:2001, ISO, 2001. 
[36] P. Jankowski-Mihułowicz and M. Wȩglarski, "Determination of 3-dimentional interrogation zone in anti-collision RFID systems with inductive coupling by using Monte Carlo method," Acta Physica Polonica A, vol. 121, no. 4, pp. 936-940, 2012.

[37] P. Jankowski-Mihułowicz and M. Wȩglarski, "Synthesis of read/ write device antenna for HF proximity range RFID systems with inductive coupling," Przeglad Elektrotechniczny, vol. 88, no. 3, pp. 70-73, 2012.

[38] P. Jankowski-Mihułowicz, "Synthesis of read/write device antenna for HF long range RFID systems with inductive coupling," Electronics, no. 8, pp. 73-77, 2010.

[39] M. H. Choi, B. D. Yang, N. S. Kim, Y. S. Kim, S. J. Lee, and K. Y. $\mathrm{Na}$, "A 13. $56 \mathrm{MHz}$ radio frequency identification transponder analog front end using a dynamically enabled digital phase locked loop," Transactions on Electrical and Electronic Materials, vol. 11, no. 1, pp. 20-23, 2010.

[40] S. Meillère, H. Barthélemy, and M. Martin, "13. 56 MHz CMOS transceiver for RFID applications," Analog Integrated Circuits and Signal Processing, vol. 49, no. 3, pp. 249-256, 2006.

[41] Y. Lam, W. Ki, and C. Tsui, "Integrated low-loss CMOS active rectifier for wirelessly powered devices," IEEE Transactions on Circuits and Systems II, vol. 53, no. 12, pp. 1378-1382, 2006.

[42] J. Lee, D. H. T. Vo, Q. Huynh, and S. H. Hong, "A fully integrated HF-band passive RFID tag IC using 0.18- $\mu \mathrm{m}$ CMOS technology for low-cost security applications," IEEE Transactions on Industrial Electronics, vol. 58, no. 6, pp. 2531-2540, 2011.

[43] K. Myny, S. Steudel, P. Vicca et al., "Plastic circuits and tags for $13.56 \mathrm{MHz}$ radio-frequency communication," Solid-State Electronics, vol. 53, no. 12, pp. 1220-1226, 2009.

[44] M. Jung, J. Kim, J. Noh et al., "All-Printed and roll-to-rollprintable 13.56-MHz-operated 1-bit RF tag on plastic foils," IEEE Transactions on Electron Devices, vol. 57, no. 3, pp. 571-580, 2010.

[45] ST-Microelectronics, "M24LR16E-R product specification," Document ID 018932, December 2011.

[46] IDS-Microchip-AG, "IDS-SL13A smart label chip with sensor for unique idenfication monitoring and data logging," IDSSL13A_SD-05. document, December 2008.

[47] X. Qing, C. K. Goh, and Z. N. Chen, "Impedance characterization of rfid tag antennas and application in tag co-design," IEEE Transactions on Microwave Theory and Techniques, vol. 57, no. 5, pp. 1268-1274, 2009. 

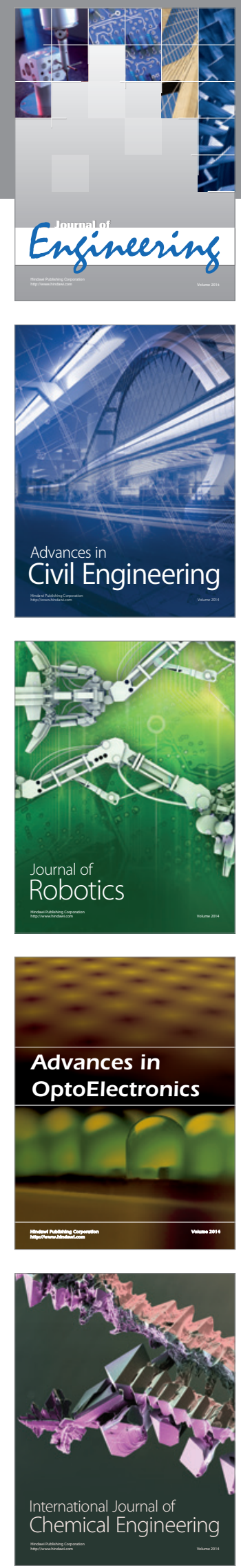

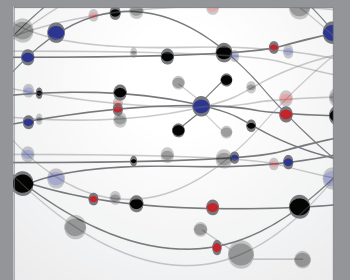

The Scientific World Journal
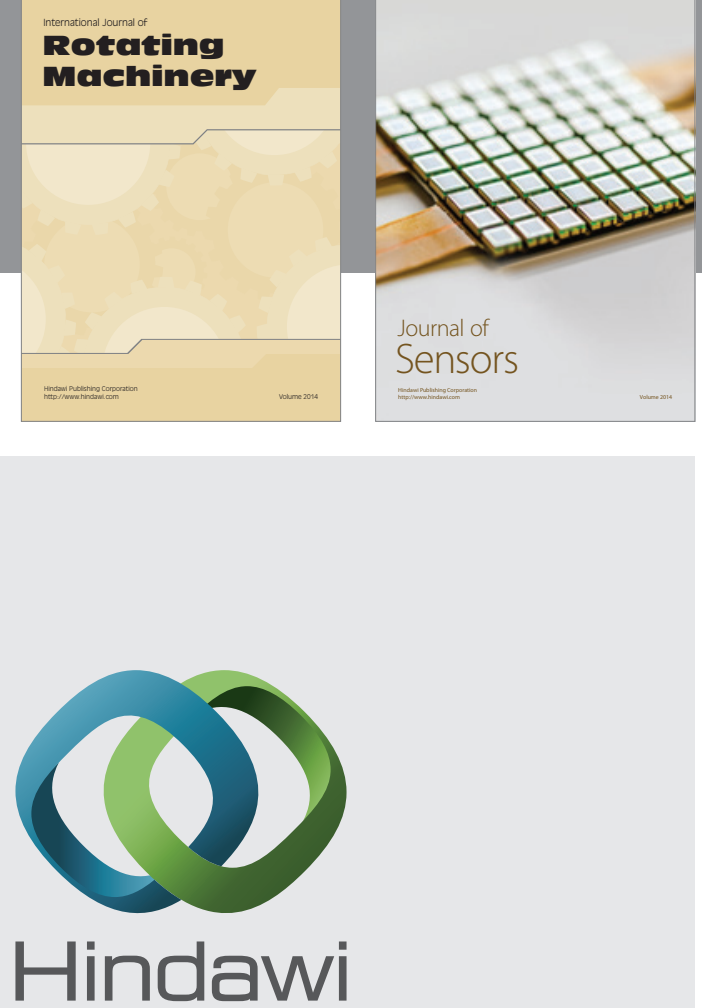

Submit your manuscripts at http://www.hindawi.com
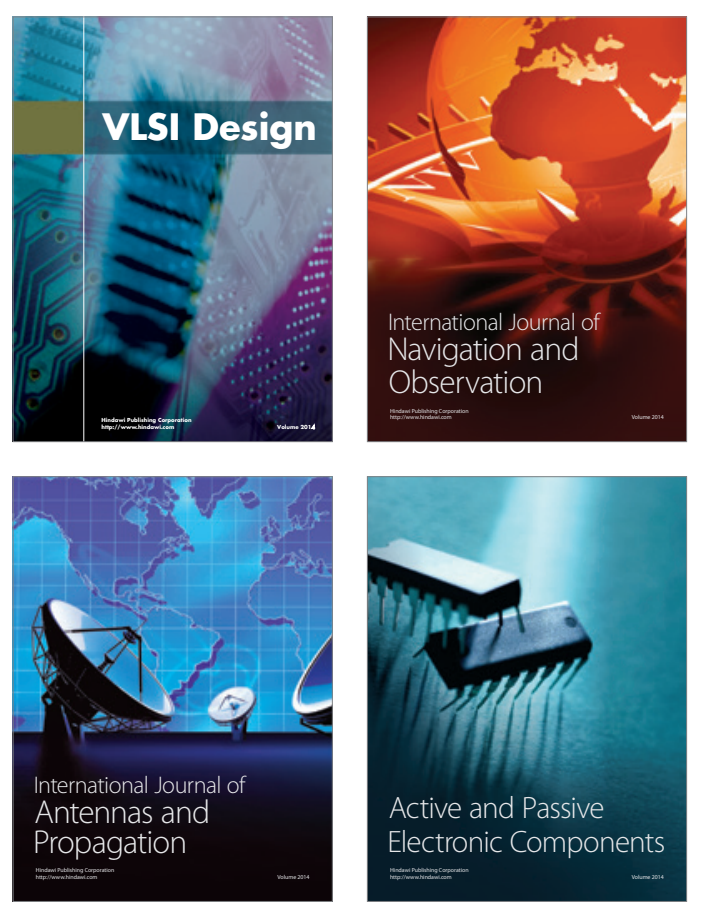
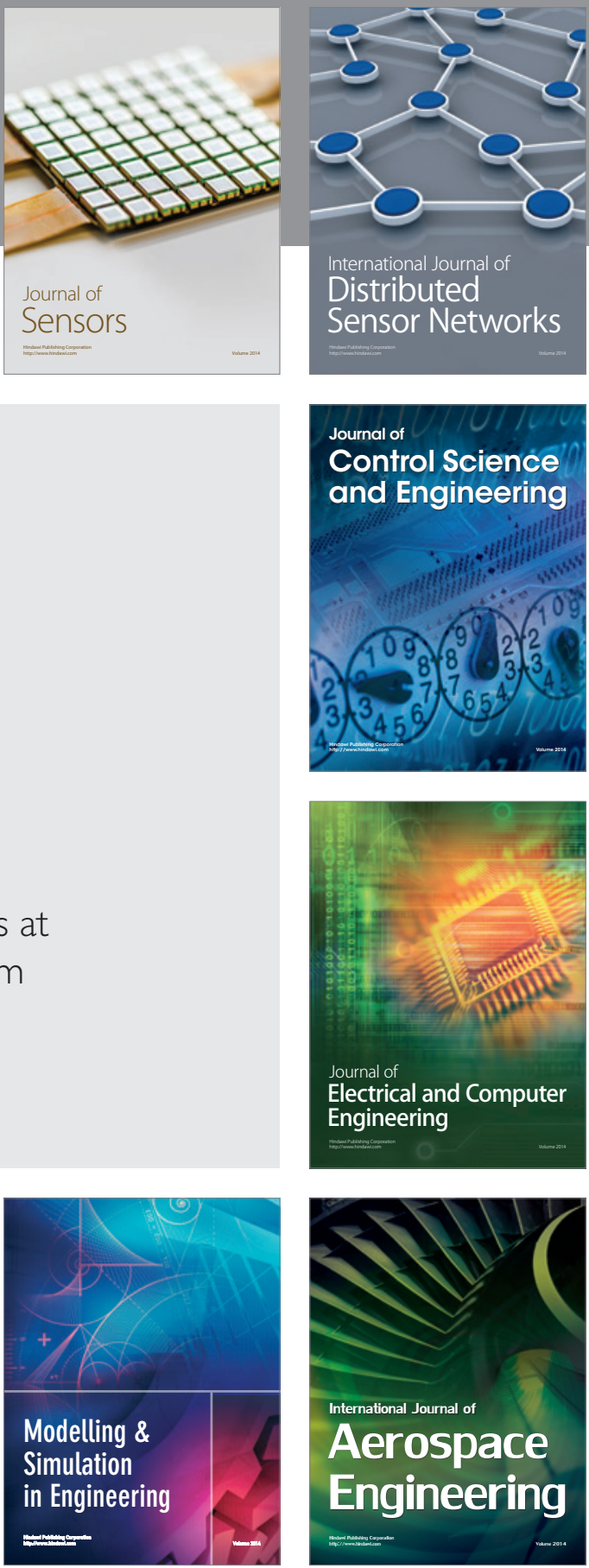

Journal of

Control Science

and Engineering
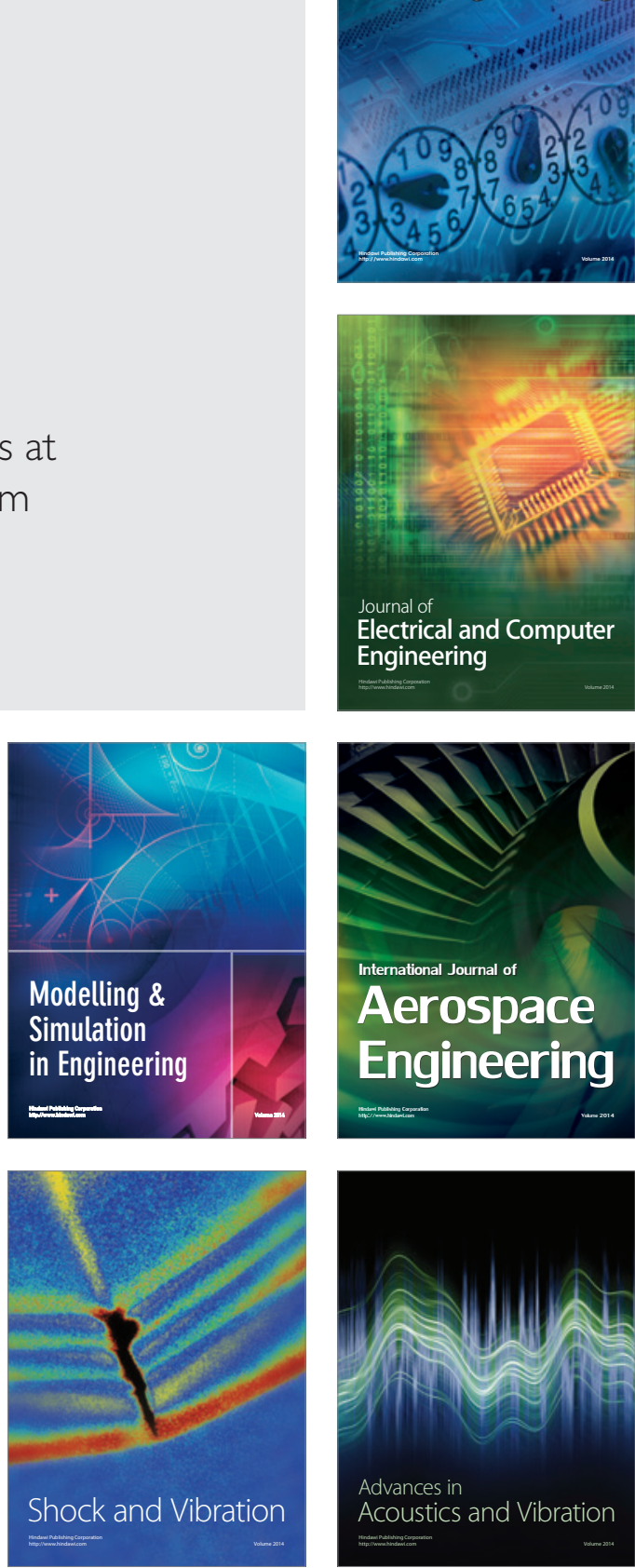